\title{
ÉPIDÉMIE DE FIÈVRE PARATYPHOİDE TRANSMISE PAR LE LAIT
}

Par MM. le Dr BRETIN

Pharmacien à Bourg (Ain) et

E. FORGEOT

Directeur des Services vétérinaires du Rhône et de l'Ain.

Aux mois d'août et dé septembre 1924, une épidémie de fièvre paratyphoïde éclatait dans la eommune de Léaz (Ain), an hameau de Grésin, s'étendait dans l'agglomération de Bellegarde-Coupy et frappait un grand nombre de personnes.

La maladie paraissait avoir commencé, dès les premiers jours d'août, sur une jeune enfant venant de Marseille, ayant présenté, d'après le médecin traitant, des troubles digestifs attribuables à l'époque de l'année. La mère, repartie à Marseille, aurait été malade à son tour. Puis la grand'mère, habitant Grésin, fut atteinte.

D'après les médecins, en quelques jours, différentes personnes sont tombées malades, avec signes intestinaux, faiblesse, ete.

La maladie (paratyphoïdè) est restée nettement Iocalisée dans le périmètre de la fontaine $A$. (Voir fig. 1.)

A Grésin, il y a eu 14 eas, avec mortalité sur deux jeunes gens et une personne âgée, morte le 28 octobre.

Vers la fin octobre, la maladie paraissait enrayée; il n'y avait pas de nouveaux cas depuis une quinzaine de jours, mais des malades étaient encore en traitement.

Vers la même époque (milieu d'août), une épidémie semblable, avee symptômes moins graves en général, est apparue à Bellegarde. En deux ou trois jours, les médecins ont eu à soigner de nombreux malades. Il y a eu ensuite une période de calme, puis de nouveaux cas sur des personnes habitant dans les maisons atteintes ou ayant soigné les premiers malades.

Les médecins traitants ont remarqué que la maladie était nettement localisée dans l'agglomération de Bellegarde, et que la zone infectée correspondait à la tournée de distribution du laitier de Grésin. La remarque avait d'ailleurs été faite que les seules personnes atteintes avaient acheté du lait près de ce laitier. Dans une maison, une femme qui avait bu du lait eru a contracté la maladie, alors que sa fille, prenant du lait bouilli, n'a pas été atteinte. Une femme qui a prélevé sur le lait et mangé de la erème fraîche fut malade, alors que sa fille alimentée avec ce lait écrémé cuit, n'a pas été touchée.

Des cas semblables ont été relevés à Coupy. Seule une malade n'a pas recu de lait de Grésin, mais elle se trouve sur la route parcourue par les personnes venant de Grésin, et a pu s'infecter par les fruits et légumes. 
L'épidémie est donc apparue à Grésin, et elle s'est transmise à Bellegarde-Coupy par le lait.

D'après l'enquête faite par M. le Commissaire spécial, il y aurait eu 80 malades environ à Bellegarde et Coupy.

Seules deux personnes, déjà âgées et malades, ont succombé.

Une seule malade aurait présenté des signes de fièvre typhoïde bien nette.

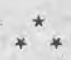

Pour comprendre l'apparition de la maladie et son extension, il faut examiner la topographie du hameau de Grésin, comprenant

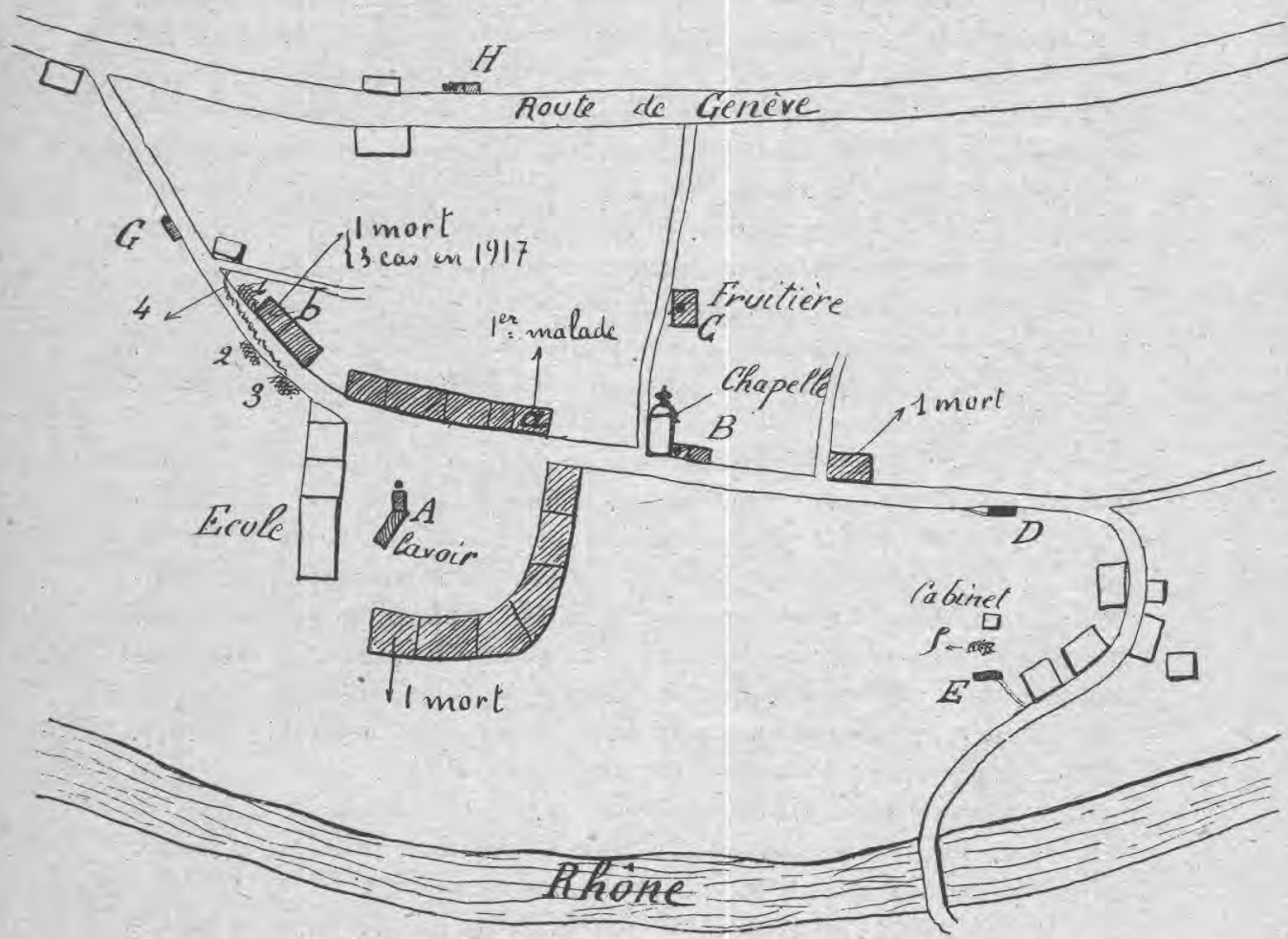

FIG, I. - Plan du hameau atteint de paratyphoïde. - A. G. H., Fontaines (eaux

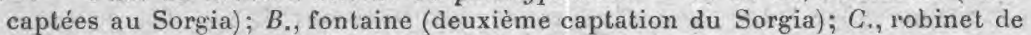
la fruitière branché sur la canalisation de la fontaine $B, ; D$. E., sources fontaines; $a$, première maison atteinte; $b$, maison où il y a eu des cas de fièvre typhoïde en rgi $7 ; 1,2,3,5$, tas de fumier; 4 , purin s'écoulant dans le chemin. - Les hachures indiquent les maisons ou il y a eu des malades.

165 habitants, son alimentation en eau, son hygiène (fumiers, eabinets, etc.).

Situé sur la pente de terrain, entre la route de Genève et le Rhône, 
c'est un hameau composé de maisons éparses, et d'autres agglomérées autour de l'école.

Le plan ci-joint, agrandissement de la carte d'état-major, et d'après un croquis rapide pris sur place, indique la topographie. La partie hachurée signale la zone infectée (fig. 1).

L'alimentation en eau est effectuée par quatre fontaines et deux sources-fontaines.

La fontaine A, située au centre d'infection, reçoit l'eau de la montagne Sorgia, où elle est captée dans un terrain de broussailles, inculte, non pâturé et ne recevant pas de fumier.

L'eau tombe d'environ 1 mètre dans un bac en pierre servant d'abreuvoir pour les animaux. En dessous de ce bac et en dénivellement de 30 à $40 \mathrm{cms}$., se trouve un autre aménagé en lavoir.

La fontaine $\mathrm{B}$, située à côté de la Chapelle, reçoit également l'eau du Sorgia, mais d'une autre captation située à environ 500 mètres de la première. Sur la même canalisation est branchée une conduite, qui amène l'eau à la fruitière $\mathrm{C}$. Elle comprend, comme la fontaine A, un bac-abreuvoir et un lavoir, situés sur le même niveau.

Une source D, aménagée avee des pierres, se trouve en contre-bas du chemin. L'eau est trouble et ne sert pas pour l'alimentation humaine. Cette fontaine est considérée comme condamnée par les personnes du hameau.

Enfin, une source $\mathrm{E}$ existe en dessous de l'agglomération des maisons de l'extrémité du village. Elle sert pour l'alimentation humaine, et les bovins y viennent boire. L'eau est claire, mais sa pollution est facile, en raison de sa situation en contre-bas des maisons. Directement au-dessus d'elle, à une trentaine de mètres, il y a un tas de fumier et des cabinets dont l'installation est rudimentaire.

Il est à remarquer que, dans ce quartier, il n'y a pas eu de malades.

Une fontaine $G$, à l'entrée du village, reçoit l'eau dans un bac en ciment; elle dessert les maisons voisines.

Enfin, une dernière fontaine $H$, se trouve sur la route de Genève. Elle comprend un bac-abreuvoir et un bac-lavoir.

Les eaux des fontaines $\mathrm{G}$ et $\mathrm{H}$ proviennent de la mème canalisation que A.

Dans le secteur de distribution des fontaines G et H, il n'y a pas eu de cas de maladie.

Fumiers. - Les fumiers sont au bord même de la rue, laquelle est en déclivité très forte. On remarquera qu'ils sont en 1, 2, 3, dans le voisinage de la canalisation des eaux; en outre, les fosses sont à même le sol où la roche, et les purins s'écoulent dans la rue en pente, pour aboutir à la place, autour de la fontaine. Il existe même en 4 une rigole d'écoulement des eaux et purins provenant des 
maisons situées en haut du chemin. Ces purins s'étalent à la surface du chemin, et les germes nocifs peuvent être transportés par les chaussures, par les animaux, etc.

Nous avons déjà signalé le tas de fumier (5) situé en haut de la source $(\mathrm{E})$.

Marche et extension de la maladie. - La maladie paraît avoir débuté sur une enfant, comme cela a été signalé plus haut, dans la maison (a), près de la fontaine. La mère aurait emporté les germes de la maladie à Marseille. La grand'mère, restant à Grésin, a été atteinte ensuite.

Cette enfant serait tombée malade quarante-huit heures après son arrivée, aurait été contaminée avant le départ de Marseille, ou en cours de route.

L'hypothèse de l'infection sur place est aussi possible. Car il faut rappeler qu'en 1917, Mme T..., dans la maison (b), a eu la fièvre typho:̈de; ses deux filles ont été également atteintes, mais elles ont été transportées à l'hôpital de Gex pour être soignées.

En raison de cette épidémie de 1917 (trois cas), la possibilité de la contamination de l'enfant est vraisemblable. En tout eas, le voisinage des fumiers $(1,2,3)$, des purins (4), permet d'envisager cette hypothèse.

D'après les renseignements, les linges de l'enfant et des malades auraient été lavés, sans ébouillantage, dans le lavoir A.

L'eau de celui-ei, contaminée, a infecté le bac supérieur où l'on doit rincer le linge. Les animaux s'abreuvant soit dans l'un, soit dans l'autre réservoir, ont pu servir de véhicule de contamination. De là, par les eaux puisées dans le bac, la transmission 's'est faite dans les maisons voisines. Le lavage des légumes crus (salades), dans ce bac, ou avec de l'eau en provenant, a pu être aussi un moyen d'infection.

Quoi qu'il en soit, la maladie a frappé un certain nombre de personnes, et notamment la femme et un enfant du laitier V..., habitant la fruitière (C).

Dans celle-ci, l'infection du lait transporté à Bellegarde s'est faite, soit par les mains du laitier, soit par les récipients, et l'on comprend l'extension de la maladie à tous les clients de ce laitier.

Un autre mode d'infection du lait mérite d'être envisagée : e'est par l'intermédiaire d'une vache laitière. Une de celles-ci a pu boire dans le lavoir (A) - les bovins recherchent d'ailleurs les eaux de savon - absorber de grandes quantités de bacilles paratyphiques, ceux-ci auront pu, cultivés dans le tube digestif, envahir les excréments, souiller la mamelle et infecter le lait. Mélangé avec la récolte du fruitier, ce lait infecté a pu constituer une excellente culture de 
bacilles paratyphiques. Le fruitier fait remarquer que son lait était meilleur que celui des autres laitiers et ne eaillait pas.

Etude bactériologique des eaux. - Les eaux ont été prélevées le 30 octobre.

Tous les bacs sont infectés, sans exception.

Toutes les sources (1) sont infectées, moins deux, et une douteuse, qui sont :

Nos 1 Source vers l'école, A.

2 Source vers l'entrée du village, du côté de Bellegarde, vers chez M. T... (Jean), G.

3 Source sur la route de Genève, $\mathrm{H}$.

Fontaines de Grésin.

Nos 1 Bac école, A, positif.

2 Bac lavoir, école, A, positif.

3 Source école, A, négatif.

4 Bac lavoir, chapelle, B, positif.

5 Bac rinçage, chapelle, $\mathrm{B}$, positif.

6 Source chapelle, B, positif.

7 Source abandonnée, D, positif.

8 Fontaine du bas, E, positif.

9 Fruitière, C, positif.

10 Source T..., G, négatif.

11 Bac route nationale, $H$, positif.

12 Source route nationale, $\mathrm{H}$, négatif.

Ces analyses permettent de différencier du paratyphique $B$ et du bacille d'Eberth.

Mesures prophylactiques. - Elles ont consisté dans les recommandations des médecins appelés auprès des malades : stérilisation de l'eau, défense de manger des fruits et des légumes crus, lavage minutieux des mains avant les repas, stérilisation des ustensiles de cuisine, des linges à l'usage des malades, désinfection des fosses d'aisance, ajournement de la rentrée des écoles jusqu'au $1^{\mathrm{er}}$ novembre, vaccination typhoïdique.

Une mesure urgente, qui n'avait pas été appliquée, était la désinfection des fontaines, abreuvoirs et lavoirs et de la fruitière, tous situés dans le foyer d'infection et ayant servi de moyens de contagion.

Nous avons recommandé au Conseiller municipal représentant le Maire la désinfection à la chaux de ces fontaines du hameau, et indiqué sur place au maçon devant. l'effectuer les précautions à prendre.

Ces fontaines ont été vidées, nettoyées, brossées et arrosées large-

(1) Par sources, il faut entendre l'eau sortant et prélevée au goulot des fontaines A, B, C, G, H et dans les deux sources D, E. 
ment, intérieur et extérieur, ainsi que le terrain environnant, au lait de chaux vive. La fruitière a été également désinfectée à la chaux, tant sur les murs que sur le sol. Tous les récipients et instruments ont été ébouillantés.

\section{**}

A Bellegarde, les conditions d'hygiène des maisons ont permis d'enrayer rapidement la maladie.

A la date du 6 octobre, après avoir consulté les médecins de Bellegarde sur l'origine de la maladie et avoir recueilli l'avis unanime que le lait de Grésin, vendu par le fruitier, était la cause de la transmission de la maladie, le Maire de Bellegarde a avisé le laitier de ne plus apporter de lait à Bellegarde.

Ce lait a servi à la préparation de fromages de Gruyère.

Aussitôt cet arrêt dans la vente du lait, les nouveaux cas de maladie ont cessé, pour reparaître une quinzaine de jours après sur des personnes en relation directe avec les malades (contamination de personne à personne).

Pour éviter la contamination par les erèmes, nous avons prié M. le Maire de Bellegarde de demander aux pâtissiers d'arrêter momentanément la fabrication et la, vente des gâteaux faits avec des crèmes non euites.

Les vaccinations ont été faites surtout par lés entérovaccins. Seuls quelques enfants ont été vaccinés par la voie sous-cutanée.

II est à remarquer dans cette épidémie que ce sont les femmes et les enfants qui ont été touchés. Le docteur Favre relève seulement trois cas de maladie sur les hommes, ayant fait la guerre et soumis aux vaccinations anti-typhiques.

Mesures à prendre. - Telles sont les mesures qui ont été prises, mais il était nécessaire d'en envisager d'autres, pour éviter l'apparition ou la réapparition de semblables épidémies :

$1^{0}$ Il importait de maintenir l'interdietion de vente du lait de la fruitière de Grésin, tant que l'épidémie ne serait pas terminée dans ce hameau. En outre, cette vente ne devra être autorisée qu'après désinfection nouvelle à la chaux de la fruitière, du sol de la chambre de réception du lait et de la cave à fromages, des instruments, et après examen bactériologique des excréments du fruitier et des membres de sa famille, pour rechercher s'il n'y a pas de porteurs de bacilles;

$2^{\circ} \mathrm{Il}$ y avait lieu de faire procéder à la désinfection des canalisations, à l'eau de Javel, en remontant aux sources ;

$3^{0}$ Il était nécessaire d'assainir le village, par la suppression des fumiers en bordure des chemins et de l'écoulement du purin sur ceux-ci et autour des fontaines. Des fosses à fumier et à purin peuvent être aménagées à peu de frais; la pierre étant sur place, quelques sacs de ciment et quelques journées de travail peuvent améliorer 
la situation. Les cabinets devraient être également construits avee fosse étanche cimentée. Il serait utile de déplacer les cabinets et fumiers situés au-dessus de la source $\mathrm{E}$ et pouvant la contaminer; $4^{\circ} \mathrm{Il}$ importait aussi de propager la vaccination antityphique dans ce hameau. Toutes les personnes devraient y ètre soumises.

La désinfection des conduites a été opérée à l'aide d'eau de Javel. Puis la fruitière a été désinfectée à nouveau; une fosse d'aisance cimentée a été construite. Un examen bactériologique ayant révélé un porteur de bacilles dans la famille du laitier, l'autorisation de vendre le lait n'a été accordée qu'après un nouvel examen négatif et après une nouvelle analyse des eaux, qui a donné un résultat négatif pour toutes les eaux des fontaines. Seules restent contaminées les deux sources $\mathrm{D}$ et $\mathrm{E}$, qui sont actuellement condamnées (1) et (2).

\section{LE PROCÈS DE LA MATIĖRE GRASSE DU LAIT} par Ch. PORCHER

- SUITE ET FIN -

Le Laboratoire officiel travaille sur des laits récemment prélevés. - Le Laboratoire agréé, que je n'entends nullement substituer à l'expert, est très bien placé pour formuler déjà une opinion plausible, à condition, bien entendu, d'avoir à sa disposition

(1) L'évolution de la paratyphoïde à Grésin nous fait apparaitre le danger de la construction des lavoirs, à eôté ou en contact avee les fontaines et bacs destinés à l'approvisionmement en eau des personnes. Ce danger devrait être signalé aux architectes, agents-voyers, etc., pour obtenir, autant que possible, la séparation et l'isolement des lavoirs publies dans les projets d'adduction d'eaux.

(2) Nous croyons utile de rapporter ici la courte discussion qui a suivi la communication du présent travail à la Société des Sciences pétérinaires de Lyon (p. 79, 1925) :

"M. F. Arloing relève l'intérêt de cette communication; la maladie a unc origine hydrique et a été transmise ensuite par le lait. Il signale l'épuration des eaux de la Saône et du Phòne, déjà signalées par Chauveau, Saturnin Arlorng, et une série de recherches sont entreprises pour connaitre l'action du bactériophage sur les coli-bacilles, les bacilles typhique et paratyphique.

« M. Porcher. - On dit généralement que les épidémies de fièvre typhoïde ou de paratyphoïde ont toujours une origine hydrique. Mais on doit noter que, parfois, il y a ensemencement direet du lait par le malade. L'origine est alors directement lactée.

"Il demande s'il y a eu des sanctions pénales, car le laitier sale devrait, avec le déeret du 25 mars 1924, être poursuivi.

"M. Fовяеот. - Des prélèvements de lait ont été faits chez ce laitier, mais, comme toujours, l'analyse n'a porlé que sur la composition chimique du lait; la recherche bactériologique n'a pas été faite. Au sujet de la laiterie, la désinfection des murs, du sol, des récipients a été opérée, ainsi que dans la salle de pesée et de réception, dans la cave à fromages. Ensuite, des eabinets étanches pour la famille du laitier ont été construits ; il est à remarquer qu'il n'y asait pas de cabinels dans cel établissement». 